\title{
REPORT OF A FATAL CASE OF LYMPHO- GRANULOMA INGUINALE FROM MENINGO-ENCEPHALITIS
}

\author{
By R. V. RAJAM, M.B.M.S. (Mad.), M.R.C.P. (E.), Venereal \\ Specialist, General Hospital, Madras
}

IT is now fairly established and accepted that the causal agent of lymphogranuloma inguinale (climatic bubo, poradenitis, lymphopathia venereum) is an ultramiscroscopic virus. The infection has been studied both on its experimental and clinical aspects. Within the past decade, a considerable amount of experimental work has been performed on animals, notably monkeys, mice and guinea-pigs. As the result of the experimental work, several facts have been elicited. (I) The varying susceptibility of different animals to intra-cranial, intracutaneous and other forms of inoculation has been studied. (2) Generalisation of the virus in the glands, liver, spleen, bone-marrow and blood after intra-cerebral inoculation has been proved. (3) In susceptible animals, monkeys and mice, intra-cranial inoculation of the virus produces a typical meningo-encephalitis with or without fatal results. The symptoms of meningo-encephalitis were those of severe meningeal irritation. "Stiffness of the neck, muscular spasm, epileptic form of convulsion, salivation, mydriasis, palsies, trembling and motor incoordination." The animals wasted and died in some from five to thirty-seven days. (4) Localisation of the virus in the central nervous system after intra-peritoneal inoculation in monkeys has been observed and the infection produced an acute infection of the posterior nerve roots and an ascending degeneration of the posterior columns of the spinal cord comparable to what happens in tabes dorsalis. According to Levaditi, the virus of lymphogranuloma inguinale in experimental intracerebral inoculation of monkeys seems to localise by preference in the mesoblastic elements of the cerebrospinal axis, meninges, blood vessels and choroidal pluxes. He calls the diseases "Neurotropic Mesodermosis," in 


\section{BRITISH JOURNAL OF VENEREAL DISEASES}

contradistinction to the other ultra-virus diseases, herpes, poliomyelitis, rabies, encephalitis, etc., which are called "Neurotropic Ectodermosis." The experimental researches so far carried out by many workers seem to point that the infection with lymphogranuloma virus is a constitutional one capable of generalisation and localisation in particular regions of the body.

On the clinical side, the several manifestations of the disease, the local adenopathy with a tendency to multiple fistulæ formation, the pseudo-elephantiasis, the constitutional symptoms of pyrexia, prostration, etc., the cutaneous and rheumatic manifestations, the occasional splenic enlargement, esthiomene in the female, ano-rectal syndrome and rectal stricture in both sexes, have been described and confirmed by many workers in the field. In human poradenitis, Nicholas (I93I) described a meningeal reaction with stiffness and intense headache which passed off in a few days. Chevalier and Barnard (I932) described a case of chronic meningeal localisation of the virus in a woman in whom the cerebro-spinal fluid showed abnormality in cell count and albumin. Medina and Vercellino, of Turin, investigated the c.s.f. of II cases of inguinal lymphogranuloma with positive Frei's reaction, in which syphilis was excluded. The c.s.f. of all except 2 were normal. In the 2 cases there was a definite increase in the cell and albumin content. The c.s.f. from these two patients gave positive skin reactions in patients suffering from lymphogranuloma. Kitagava, of Japan, in 1934, investigated the c.s.f. of a number of cases of lymphogranuloma and found definite abnormalities in a few of the cases. David and Lorin (1936), in discussing the extra-genital lesions of lymphogranuloma, mentions an interesting case of ano-rectal syndrome in a woman aged thirty-two with positive Frei's test, in which the disease processes had extended to the colon and the patient suffered from repeated epileptic form convulsions. He suggests the possibility of the latter condition being caused by a lymphogranulomatous encephalitis, and the relation of lymphogranuloma inguinale to meningo-encephalitis of unknown origin should always be suspected. Few post-mortem records of these diseases are available. Reichle and Connor, in 1935, described the post-mortem findings of a case of lymphogranuloma in which there was widespread involvement of the retro- 


\section{CASE OF LYMPHOGRANULOMA INGUINALE}

peritoneal lymph-nodes, hip joint, adrenals and kidney. In view of this ubiquitous pathology, clinicians and specialists should always be on the look out for the extragenital and extra-rectal manifestations of the infection.

As far as the writer is aware from the available literature no fatal human case of acute meningo-encephalitis of suspected lymphogranulomatous ætiology has been reported, and the following case is presented for discussion.

A male, aged twenty-seven, came to the venereal department of the General Hospital, Madras, on February 6th, I935, with inguinal lymphogranuloma of three weeks' duration.

Condition on Admission.-The patient was looking ill, with a temperature of $\mathrm{IOO}^{\circ} \mathrm{F}$. There was no sore on the genitals or urethral discharge. There was a large elongated swelling in the right groin, consisting of enlarged, hard, tender, matted glands. The skin over the swelling was thickened, œdematous and rugose. The iliac glands on the same side were also enlarged and tender. No other cutaneous or mucous membrane lesion was observed.

Investigations.-Blood: Both the Kahn and Wassermann reactions were negative.

The Frei's intra-dermal test was strongly positive with a tendency to pustular reaction. The patient attended the clinic for three days and defaulted. No other treatment was given to him except hot fomentations to the groin during the period he was attending the hospital.

Nine days later the patient was brought to the hospital in a comatose condition, and the history elicited from his relations was as follows:

On February I2th, I935, an operation was performed on him by a quack for the bubo, and the patient was apparently all right for three days. At 3 a.m. on February I5th, I935, he developed high fever, started behaving in a queer manner, which was rapidly followed by generalised convulsions. From the first fit he became unconscious, and the patient was brought to the hospital at 5 a.m. on the same day. According to the report of the relations, no injections of any kind were given to the patient.

Condition on Admission on February I5th, I935.-The patient was deeply comatose with conjugate deviation of the head and eyes to the left side. While he was being examined he had a fit. Rectal temperature I04.4. 


\section{BRITISH JOURNAL OF VENEREAL DISEASES}

Respiration was of the Cheyne-Stokes' type. Pulse I38, volume and tension poor. Pupils react very sluggishly to light and unequal in size. The tendon jerks of the lower extremities were extra brisk. The plantar reflex was extensor on both sides. Kernig's sign positive. No definite paresis or paralysis of the limbs. Bladder distended. Lock-jaw absent. On the right groin there was a deep, dirty operation wound 3 inches long, exposing the half-cut lymph glands oozing pus. There was still evidence of the positive Frei's skin reaction in the form of a pigmented circular area with a central scab.

Investigations. - The examination of the blood-smear was negative to malarial parasites, but showed a definite leucocytosis ( 16,000 principally polymorpho-nuclear leucocytes). Urine contained a faint trace of albumin, but no casts or sugar. Lumbar puncture was performed, and the fluid was clear, but under great pressure. Examination of the cerebro-spinal fluid revealed the following : Cells 136 per cubic micromillimetre, of which 90 were polymorphs and the rest lymphocytes. Albumin $95 \mathrm{mgm}$. per cent. Globulin $25 \mathrm{mgm}$. per cent. Wassermann reaction negative in two dilutions. Goldsol. test 2552533433. The cultures of blood and c.s.f. were sterile. $O^{\circ} I$ c.c. of the undiluted crebro-spinal fluid drawn from the patient was injected intradermally on two lymphogranuloma patients and a positive reaction was obtained in both of them.

Treatment.-In spite of repeated lumbar puncture, continuous oxygen inhalation and intravenous injection of hypertonic saline, the patient was getting repeated epileptic form convulsions and died at 8 p.m. on the same day, seventeen hours after the commencement of the attack. To the great regret of the writer, permission for even a limited post-mortem examination could not be obtained from the relatives.

Commentary.-In the case under report, the clinical and serological examination of blood and cerebro-spinal fluid were negative to syphilis. No history of any injection of an arsenical drug given prior to the fatal attack was elicited either from the relations or from the quack who operated on him. Tetanus was ruled out in the absence of the characteristic spasms, facies and lock-jaw. It appears to me that this is a case of meningoencephalitis caused by the virus of lymphogranuloma. 


\section{CASE OF LYMPHOGRANULOMA INGUINALE}

On the analogy of the possibility of the experimental production of the disease in animals, there is no reason why such a localisation of the virus in the central nervous system should not occur in the case of human lymphogranuloma. As mentioned before, the symptoms of meningeal irritation and localisation in human cases have been observed by others, and it is possible that a particularly virulent strain of the virus, as in the case under discussion, could have disseminated from the blood-stream and resulted in a fatal localisation in the central nervous system. Our diagnosis of lymphogranulomatous meningo-encephalitis in this case would have received added confirmation if a post-mortem examination of the brain and meninges of the patient had been available for experimental animal inoculation.

Conclusion.-A case is presented here of lymphogranuloma inguinales who developed a fatal and fulminating meningo-encephalitis during the fourth week of the disease, in whom the Frei's intradermal reaction was strongly positive, and whose cerebro-spinal fluid gave a positive reaction in two other lymphogranuloma patients. Evidence of other venereal diseases was absent. Coma due to other causes were excluded as far as possible.

From the experiment, and to a less extent from the clinical side, it appears that the virus of the disease may be productive of an as yet undetermined pathology. It is not improbable that certain strains of the organism may have a special tendency to localisation in various parts of the body, particularly the central nervous system.

\section{REFERENCES}

(I) Stannus, H. S. : “ A Sixth Venereal Disease" (I933).

(2) Stannus, H. S. : "Poradenolymphitis," Tropical Diseases Bulletin, Vol. 31, No. 7 (I934).

(3) Thompson, R. M. : "Lymphopathia Venereum," Journal of the American Medical Association, Vol. 106, No. 22.

(4) David, V. C., and LoRING, M.: "Extragenital Lesions of Lymphogranuloma Inguinale," Journal of the American Medical Association, Vol. 106, No. 22. 